\title{
Transient band structures in the ultrafast demagnetization of ferromagnetic gadolinium and terbium
}

\author{
Martin Teichmann, Björn Frietsch, Kristian Döbrich, Robert Carley, and Martin Weinelt \\ Freie Universität Berlin, Fachbereich Physik, Arnimallee 14, 14195 Berlin, Germany \\ and Max-Born-Institut, Max-Born-Strasse 2a, 12489 Berlin, Germany
}

(Received 8 November 2013; revised manuscript received 19 December 2014; published 21 January 2015)

\begin{abstract}
We compare the laser-driven demagnetization dynamics of the rare earths gadolinium and terbium by mapping their transient valance band structures with time- and angle-resolved photoelectron spectroscopy. In both metals, the minority and majority spin valence bands evolve independently with different time constants after optical excitation. The ultrafast shift of the partially unoccupied minority spin bulk band to higher binding energy and of the majority spin surface state to lower binding energy suggests spin transport between surface and bulk. The slower response of the fully occupied majority spin band follows the lattice temperature and is attributed to Elliott-Yafet type spin-flip scattering. Terbium shows a stronger and faster decay of the exchange splitting, pointing to ultrafast magnon emission via $4 f$ spin-to-lattice coupling.
\end{abstract}

DOI: 10.1103/PhysRevB.91.014425

PACS number(s): 75.78.Jp, 78.47.-p, 71.20.Be

\section{INTRODUCTION}

Laser-driven ultrafast magnetization dynamics is the subject of intense ongoing experimental and theoretical studies. For metallic systems, these efforts encompass both elemental itinerant [1-4] and rare-earth [5-7] ferromagnets, as well as alloys and layered structures [8-12]. Besides being technologically interesting, new experimental results challenge our understanding of magnetism [11,13]. Since the pioneering magneto-optical Kerr effect (MOKE) study on nickel by Beaurepaire et al. [14], it has been confirmed by several experimental techniques that laser-driven demagnetization occurs in the itinerant ferromagnets within 0.3 ps [4,15-18]. In contrast, for the rare earths gadolinium and terbium, slower decay constants of $0.75-1.0$ ps have been reported $[5-7,19]$. The different dynamics in the itinerant and rare-earth ferromagnets are believed to be important for all-optical magnetic reversal observed in the ferrimagnetic $\mathrm{FeCoGd}$ alloy $[8,13]$. The magnetization dynamics was explained by the interplay between the material-specific decay constants and the angular momentum transfer among the $\mathrm{Fe}$ and $\mathrm{Gd}$ sublattices [20,21]. This ultrafast angular momentum transfer was experimentally confirmed for CoGd and CoTb ferrimagnets [12], where the initial demagnetization is driven by the local transfer of angular momentum between the exchangecoupled sublattices while maintaining the total angular momentum.

Despite the significant progress in characterizing ultrafast magnetization dynamics [22], the underlying microscopic processes are still debated. Koopmans et al. suggested that the different time scales observed in ultrafast demagnetization can be universally described by Elliott-Yafet type (EY) electronphonon spin-flip scattering [23]. Subsequently, it was shown that the microscopic three-temperature model model (M3TM) can describe the magnetization dynamics for a wide range of starting temperatures and excitation fluences [18,24]. Variations of the M3TM have been discussed, including magnon emission and Coulomb interaction [25,26]. Nevertheless, the model was challenged by first-principles calculations, which found that spin mixing of the valence bands and the concomitant spin-flip probability upon electron scattering are too small to account for femtosecond demagnetization [27]. The theory considered a rigid band structure. However, pioneering photoemission experiments on laser-excited $\mathrm{Ni}$ indicated that the exchange splitting reduces on the femtosecond time scale [16]. These results were then substantiated by our study on Gd [7]. Two calculations account for this dynamic reduction in exchange splitting but have come to different conclusions on the enhancement and thus importance of EY spin-flip scattering [28,29]. A very recent study reports on stronger EY scattering in a multilayer structure of $\mathrm{CoPt}$ as compared to the pure Co film, which was attributed to spin-orbit coupling at the interface [24].

As a counter-concept Battiato et al. proposed superdiffusive spin transport as the leading process and explored it for $\mathrm{Ni}$ [30], for which theory predicts a large spin asymmetry of the excited electron lifetimes [31]. Follow-up studies suggest that for $\mathrm{Ni}$ multilayer systems both spin-flip scattering and transport are operative on the femtosecond time scale [9,18,32]. X-ray scattering experiments in nanoscale $\mathrm{CoPd}$ and $\mathrm{CoPt}$ domain networks revealed shortened demagnetization times compared with pure Co metal [33] and domain wall changes [34], which were explained by spin currents between neighboring domains $[33,34]$. There is likewise evidence that spin currents on the nanoscale drive the magnetic reversal in the FeCoGd alloy [10].

While the itinerant ferromagnets, in particular $\mathrm{Ni}$, have been extensively studied, more data on the lanthanides are required to distinguish between the microscopic mechanisms determining ultrafast magnetization dynamics. In contrast to the itinerant $3 d$-ferromagnets, the equilibrium magnetism of the lanthanides is dominated by the $4 f$ electrons, which are strongly localized but coupled via indirect exchange mediated by the $(5 d 6 s)^{3}$ valence electrons [35], Therefore, besides transport and spin-flip scattering in the valence bands, the valence- $4 f$ exchange and $4 f$-spin-lattice coupling can contribute to the magnetization dynamics [5]. Among the heavy lanthanides, Gd is special in that it has a half-filled $4 f$ shell with vanishing orbital angular momentum $\left(4 f^{7}, L \approx 0\right)$, suppressing direct coupling of the $4 f$ spin system to phonons. This channel is present in $\mathrm{Tb}\left(4 f^{8}, L=3\right)$. This argument was previously brought forward to explain the different 
magnetization dynamics of $\mathrm{Gd}$ and $\mathrm{Tb}$ observed by $\mathrm{x}$-ray magnetic circular dichroism (XMCD): decay constants of about 40 and 8 ps, respectively [5,19].

In this paper, we compare the transient valence bandstructures of $\mathrm{Gd}$ and $\mathrm{Tb}$. After excitation with a femtosecond laser pulse the binding energies of the exchange-split $\Delta_{2}$-like $\Sigma$ valence bands [36] change to values different from the ones acquired by thermal heating. We observe a faster response of the partially unoccupied minority spin valence band than its completely occupied majority counterpart. The combination leads to a drop of the exchange splitting, which we show can not be described by spin-flip scattering alone. In addition the occupied majority surface state shifts towards the Fermi energy and becomes partially depopulated [37]. Thus spin-transport and EY spin-flip scattering are both candidate mechanisms for ultrafast demagnetization of the rare earths. Moreover, $\mathrm{Tb}$ shows a faster and stronger response to optical excitation than $\mathrm{Gd}$, which we attribute to ultrafast $4 f$ spin-to-lattice, i.e., magnon-phonon coupling.

\section{EXPERIMENT}

All experiments were performed at a base pressure of $3 \times$ $10^{-11}$ mbar in a two-chamber ultra-high-vacuum system for surface preparation and angle-resolved photoemission spectroscopy (ARPES). Single crystalline $\mathrm{Gd}(0001)$ or $\mathrm{Tb}(0001)$ films were prepared by evaporation onto a W(110) single crystal to a thickness of 5-10 nm $(\approx 18-36$ monolayers $)$ $[7,36]$. The pressure during evaporation was $1 \times 10^{-10} \mathrm{mbar}$. Film thickness was controlled by recording the evaporation rate with a quartz microbalance. To improve the crystallinity after evaporation, $\mathrm{Gd}$ and $\mathrm{Tb}$ films were annealed to 650 and $880 \mathrm{~K}$, respectively. The surface quality was monitored using low-energy electron diffraction and ARPES. The samples were cooled with liquid nitrogen to a temperature of $\approx 100 \mathrm{~K}$. For time-resolved ARPES, we use a high-order harmonic generation beamline described in detail in Ref. [38]. The driver laser is a commercial Ti:Sapphire amplifier (KMLabs RedDragon), with a wavelength of $785 \mathrm{~nm}$ at a repetition rate of $10 \mathrm{kHz}$ and a pulse duration of $40 \mathrm{fs}$. In the pump-probe experiment, the valence electronic system was excited with the laser fundamental, $s$-polarized, and stretched to $300 \mathrm{fs}$ to reduce space charge [38]. To evaluate the absorbed fluence of the pump pulse, we measured the power of the incident and reflected beam and recorded the spot size on a scattering surface at the sample position with a CCD camera. This procedure leads to an uncertainty in absolute values of the absorbed fluence of about $18 \%$. After a variable delay, the sample was probed with a $p$-polarized XUV pulse with a duration of $100 \mathrm{fs}$, produced by high-order harmonic generation in argon. We selected an XUV wavelength of $35.6 \mathrm{eV}$ and a bandwidth of $150 \mathrm{meV}$ using a toroidal grating monochromator. The pump and probe pulses impinge on the sample nearly collinearly at an angle of $60^{\circ}$ to the surface normal. Photoemitted electrons were detected with an image-type hemispherical analyzer (SPECS Phoibos 100). At the chosen photon energy of 35.6 $\mathrm{eV}$, the Brillouin zone is cut at a $\Gamma$ point [39], and we map valence bands with parallel momentum $k$ in the $\Gamma-M$ direction [36].

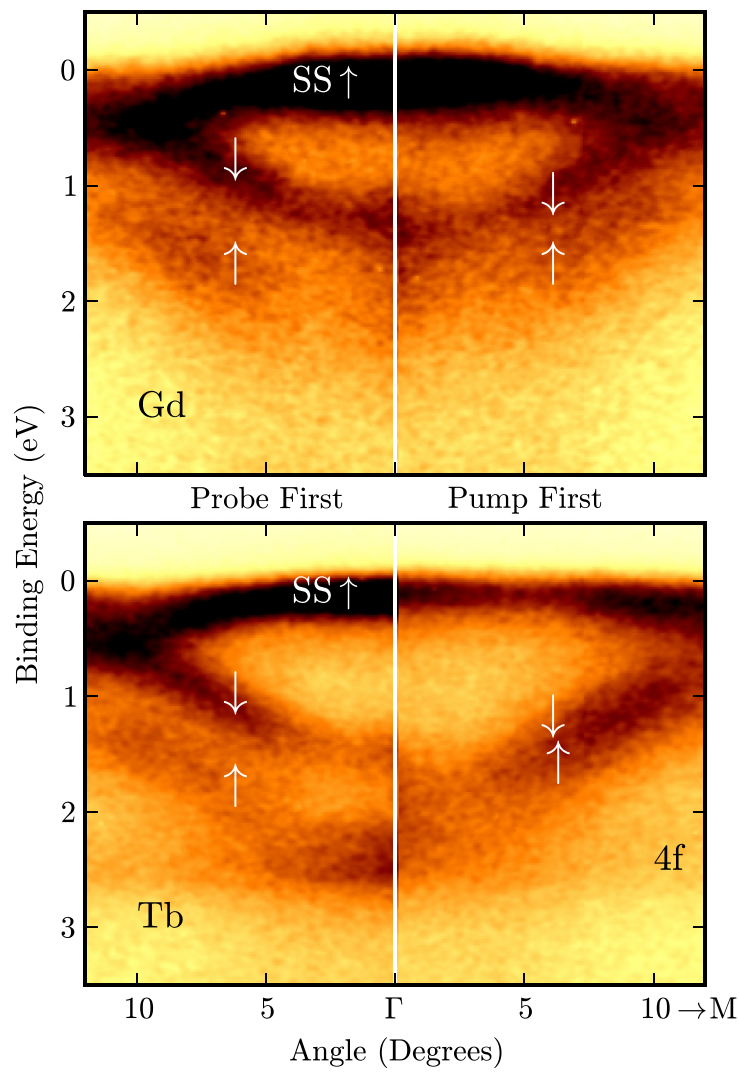

FIG. 1. (Color online) ARPES data for Gd (top) and Tb (bottom). Both panels are split at the $\Gamma$ point. The left side shows the exchange split minority and majority spin valence bands ( $\downarrow$ and $\uparrow)$ for the sample at $100 \mathrm{~K}$ before excitation. The right side represents minimal exchange splitting at a pump-probe delay of $4 \mathrm{ps}$. Absorbed pump fluences for $\mathrm{Gd}$ and $\mathrm{Tb}$ are $5.5 \pm 1$ and $3.6 \pm 0.7 \mathrm{~mJ} / \mathrm{cm}^{2}$, respectively. SS $\uparrow$ is the majority spin surface state and $4 f$ marks the Tb multiplet component $S_{7 / 2}$.

\section{RESULTS}

\section{A. Transient band structure}

The top and bottom panels of Fig. 1 show four plots of ARPES data before and after optical excitation for $\mathrm{Gd}$ and $\mathrm{Tb}$, respectively. Overall, the valence band structures of both materials are very similar. Both data sets are dominated by photoemission from the occupied majority spin surface state (SS $\uparrow$ ) close to the Fermi edge $E_{\mathrm{F}}=0[37,40]$. Below that, lie the $\Delta_{2}$-like $\Sigma$ minority and majority spin valence bands ( $\downarrow$ and $\uparrow$ ), which are exchange split if the sample is cooled below the Curie temperature $T_{\mathrm{C}}(293 \mathrm{~K}$ for $\mathrm{Gd}$ and $220 \mathrm{~K}$ for $\mathrm{Tb}$ ) $[36,39,41]$, as depicted on the left hand side of Fig. 1 (probe first). The right-hand side shows ARPES data 4 ps after optical excitation (pump first). At this delay, the exchange splitting $\Delta E_{e x}$ reaches its minimum and while it decreases in $\mathrm{Gd}$, it nearly vanishes for $\mathrm{Tb}$. In parallel, the surface state $\mathrm{SS} \uparrow$ shifts to lower binding energy and shows reduced intensity [37,40]. In Tb, the $4 f^{8} S_{7 / 2}$ multiplet component gives rise to a nondispersing final state, which coincides with the bottom of the majority spin valence band around the $\Gamma$ point [42]. 


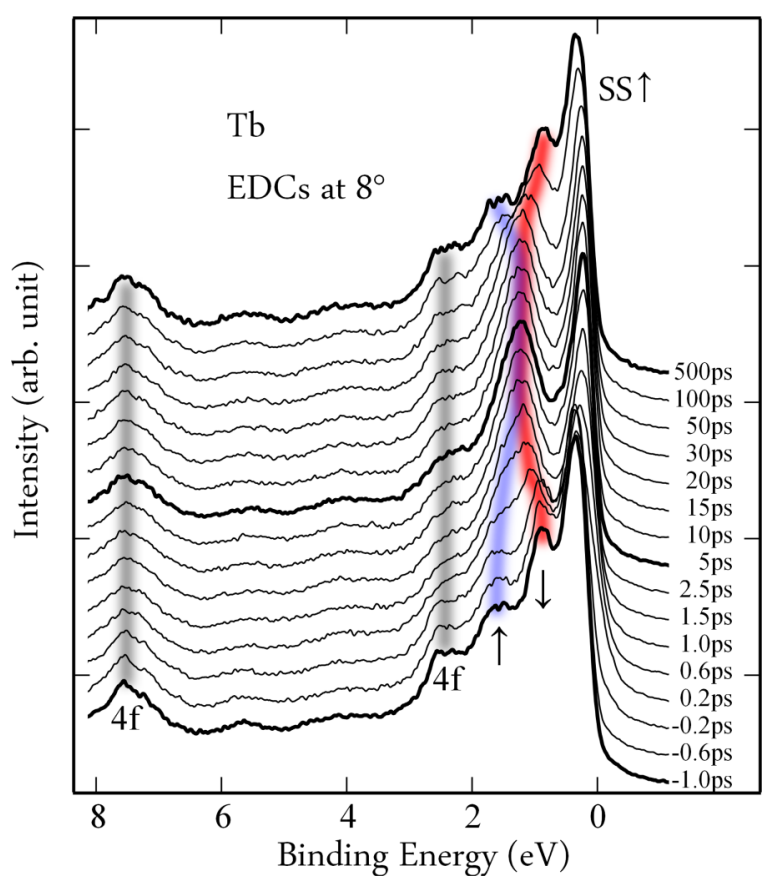

FIG. 2. (Color online) Energy distribution curves for Tb extracted at an emission angle of $8^{\circ}$ in Fig. 1. The delay between the IR pump and VUV probe pulse increases from bottom to top. Note that the delay steps are not constant. SS $\uparrow$ is the occupied Tb majority spin surface state, $\downarrow$ and $\uparrow$ mark the $\Delta_{2}$-like $\Sigma$ minority and majority spin valence bands, and $4 f$ denotes components of the $\mathrm{Tb}$ $4 f$ multiplet. Upon optical excitation at zero pump-probe delay, the $\mathrm{SS} \uparrow$ shifts to lower binding energies and the exchange splitting of the valence bands decreases. At larger pump-probe delay, the spectra reveal the recovery of the surface and bulk exchange splitting.

Figure 2 illustrates the dynamics of the $\mathrm{Tb}$ valence bands as a function of delay between the IR pump and the VUV probe pulse. The energy distribution curves (EDCs) have been extracted at an emission angle of $8^{\circ}$ to separate the majority spin valence band from the Tb $4 f^{8} S_{7 / 2}$ multiplet component. Within the first 2 ps after optical excitation, the majority spin surface state ( $\mathrm{SS} \uparrow$ ) shifts towards the Fermi level and we observe a nearly symmetric convergence of the minority and majority spin bands ( $\downarrow$ and $\uparrow$ ), as indicated by the red and blue highlighters in Fig. 2. Thereafter, the peak positions remain nearly constant until the recovery of the exchange splitting sets in at about $30 \mathrm{ps}$. Similar spectra for Gd, though extracted at an emission angle of $0^{\circ}$, can be found in Ref. [7].

To reliably extract the peak positions at the center of the Brillouin zone, $\Gamma$, we have fitted the entire momentum-energyspectrum in Fig. 1 to a model function, including SS $\uparrow$, as well as $\downarrow$ and $\uparrow$ components of the valence bands. Each band is a Lorentzian in the energy direction and a parabola in the momentum direction, with an effective electron mass for the dispersion. The angular asymmetry seen in the data is modeled by a cosine modulation, representing the angular dependence of the dipole transition matrix element. The model function is multiplied with a Fermi-Dirac distribution to determine the electronic temperature $T_{\mathrm{e}}$ of the sample [43]. Finally, a linear and an integral background are included. The comprehensive fit allows us to extract the binding energies of the valence bands

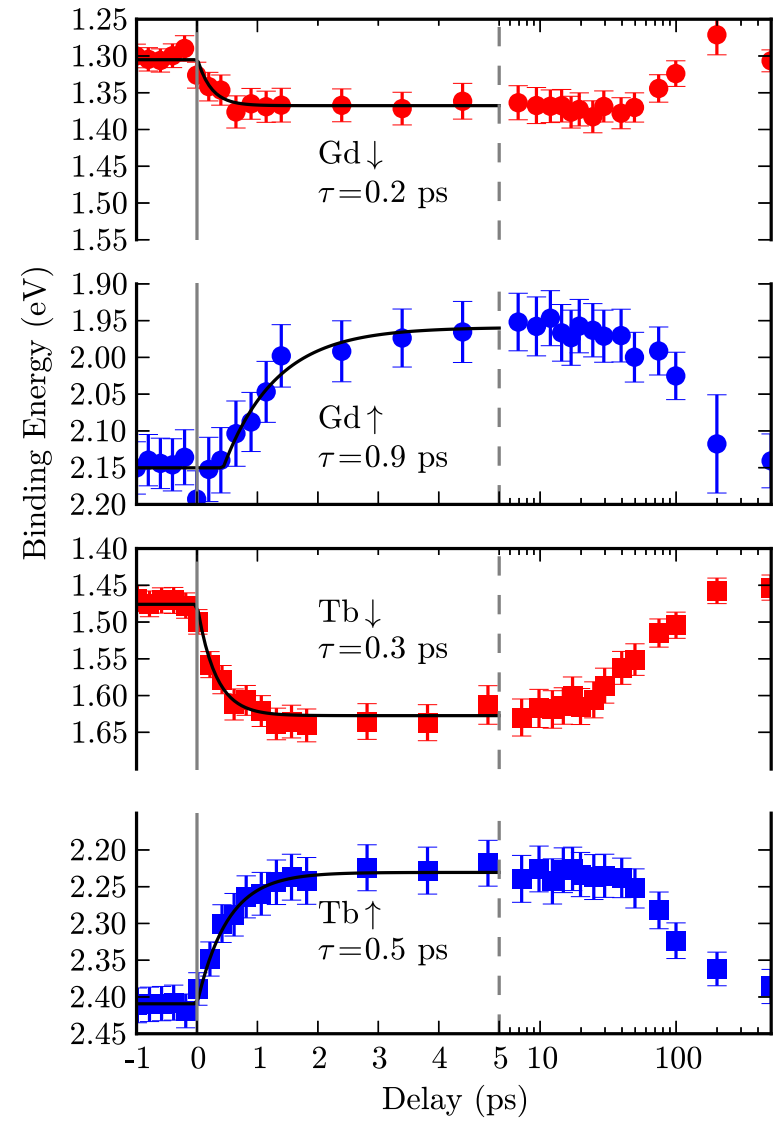

FIG. 3. (Color online) Binding energies of the minority ( $\downarrow$, red) and majority ( $\uparrow$, blue) spin bands at $\Gamma$ as a function of pump-probe delay, for Gd (circles) and $\mathrm{Tb}$ (squares). Solid lines are exponential fits with time constant $\tau$ indicated in the figure. The time axis is linear for short times, but logarithmic for longer times. Error bars show two standard deviations, estimated from the fit.

( $E \uparrow$ and $E \downarrow$ ) as a function of pump-probe delay. For $\mathrm{Gd}$, the global fit reveals a small $k$-dependence of the band dynamics. This may be related to a $k$-dependent hybridization of the $4 f$ and $5 d 6 s$ orbitals and thus a $k$-dependent intra-atomic exchange [44] and/or the $k$-dependent mixing of majority and minority spin valence bands described in Ref. [35]. Please note that the degree of spin mixing of the valence bands affects the EY spin-flip scattering probability. These very details do not affect our general conclusions. Thus we concentrate in the following on the dynamics at the Brillouin zone center $\Gamma$. The band positions at $k=0$ are displayed in Fig. 3 for Gd (circles) and $\mathrm{Tb}$ (squares). In the first $1.5 \mathrm{ps}$ after laser excitation minority and majority spin bands converge, reducing the exchange splitting. After a plateau with nearly constant binding energies, starting at about $20-40 \mathrm{ps}$, we see the thermal recovery of the valence-band positions upon cooling of the irradiated spot.

The temperature dependence of the valence band bindingenergies for $\mathrm{Gd}$ and $\mathrm{Tb}$ have been studied by ARPES across the phase transition [41]. Between $100 \mathrm{~K}$ and $T_{\mathrm{C}}$, the binding energy of the Gd majority spin band shifts about twice as much as its minority counterpart. Recent calculations of the Gd band structure by Sandratskii [35] model 
a reduced magnetization assuming disorder, i.e., tilting of the $4 f$ moments. They compare calculations of Gd bulk with the quantized band structure of a 12-monolayer-thick Gd slab. The slab calculations confirm that the valence bands probed in photoemission are predominantly located in the inner part of the slab [35]. At $T=0$, where spin mixing is absent, the lower state is of majority spin character, whereas the upper state is of the spin minority type. Finite temperature is modeled by noncollinear $4 f$ spins. This yields an asymmetric closing of the exchange splitting as a result of the perturbation of the bulk electronic structure by truncation at the surface, while the exchange splitting closes symmetrically for bulk Gd [35]. In contrast, when heating $\mathrm{Tb}$ towards $T_{C}$, minority and majority spin bands show a similar shift in ARPES so that the exchange splitting of Tb closes symmetrically [41].

We find comparable behavior upon optical demagnetization as depicted in Fig. 3. Also on the ultrafast time scale, the shift of the valence bands is asymmetric in Gd but symmetric in $\mathrm{Tb}$. The shift of the valence bands is as expected from their temperature-dependent behavior in thermal equilibrium. However, in the first $1.5 \mathrm{ps}$ after pumping the dynamics of the minority and majority spin bands exhibit clearly different time constants $\tau$ (compiled in Fig. 3). In addition, we observe a delayed response of the Gd majority compared to the minority spin band. The slight difference of this delay compared to Ref. [7] is still within error bars.

\section{B. Exchange splitting}

At present there is no model available that describes the individual dynamical response of the valence bands of a ferromagnet to optical excitation. However, the M3TM allows us to calculate the decay of the exchange splitting $\Delta E_{\mathrm{ex}}=E \uparrow-E \downarrow$ in terms of EY electron-phonon spin-flip scattering [23]. Figure 4 depicts the initial dynamics of the exchange splitting for $\mathrm{Gd}$ (circles) and $\mathrm{Tb}$ (squares) calculated from the data in Fig. 3. The inset shows the transient electronic temperature $T_{e}$ derived from the fit of the Fermi edge. The electronic temperature shows a delay-dependent offset due to the large intensity of the SS $\uparrow$ surface state and a small space-charge-induced transient broadening of its linewidth. This pump-induced effect is mainly seen for $\mathrm{Gd}$, since for one we use a higher pump fluence and for another Gd has a significantly smaller work function than $\mathrm{Tb}$ (3.72 versus $4.95 \mathrm{eV})[45,46]$.

The rise of $T_{e}$ by about $5000 \mathrm{~K}$ in $\mathrm{Gd}$ and $2500 \mathrm{~K}$ in $\mathrm{Tb}$ reflects the different absorbed fluences of the pump pulse of $5.5 \pm 1$ and $3.6 \pm 0.7 \mathrm{~mJ} / \mathrm{cm}^{2}$, respectively. The transient of $T_{e}$ for $\mathrm{Gd}$ is in accord with previous simulations for $\mathrm{Gd} / \mathrm{W}(110)$ (see Fig. 1 in Ref. [47]). Via the electronic temperature and the exchange splitting, we have measures of two of the relevant subsystems in the ferromagnet: exited carriers and spins of the valence bands [14]. The fit of the M3TM to the exchange splitting and electronic temperature is shown as solid lines in Fig. 4. The M3TM predicts an analytic expression for the dynamics of the exchange splitting from known material constants and band-structure calculations based on the Weiss mean-field description (see Ref. [23] and

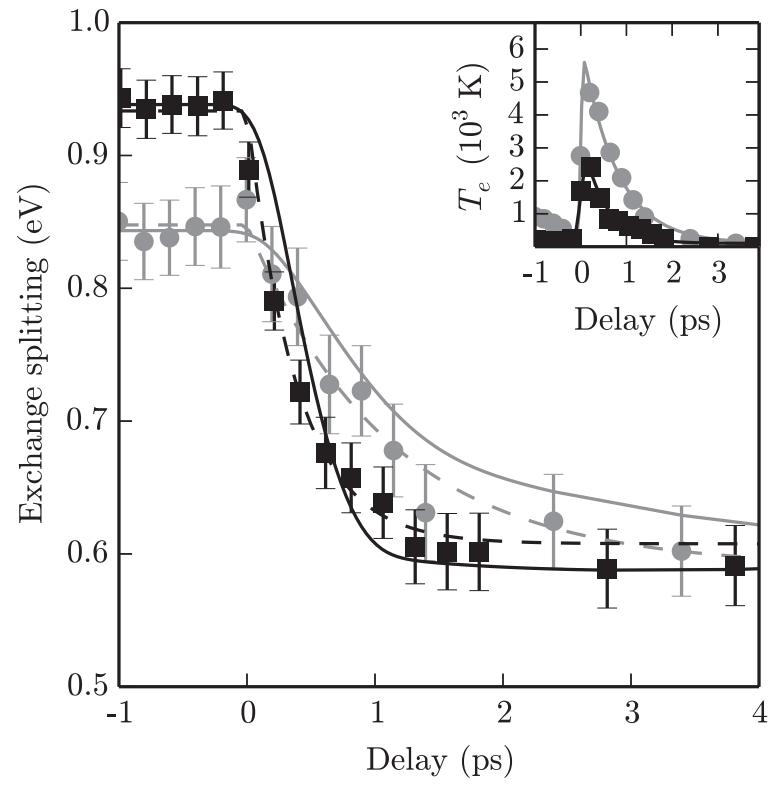

FIG. 4. The time evolution of the exchange splitting for $\mathrm{Gd}$ (circles) and $\mathrm{Tb}$ (squares), with a fit to the M3TM (full line) and a simple exponential decay (dashed line). The inset shows the electronic temperatures $T_{\mathrm{e}}$ from the broadening of the Fermi edge. The electronic temperature has a delay-dependent offset due to the tail of the surface state $\mathrm{SS} \uparrow$.

the references therein):

$$
\frac{d\left(\Delta E_{\mathrm{ex}}\right)}{d t}=\frac{\Delta E_{\mathrm{ex}}}{\tau_{R}} \frac{T_{p}}{T_{C}}\left(1-\frac{\Delta E_{\mathrm{ex}}}{2 k_{B} T_{C}} \operatorname{coth} \frac{\Delta E_{\mathrm{ex}}}{2 k_{B} T_{e}}\right) .
$$

The characteristic time constant $\tau_{R} \propto \mu / \gamma^{2} T_{\mathrm{C}}^{2}$ contains the magnetic moment $\mu=7.55(9.34) \mu_{\mathrm{B}}$, the electronic specific heat $\gamma=4.48(3.71) \mathrm{mJ} /\left(\mathrm{mol} / \mathrm{K}^{2}\right)$, and the Curie temperature $T_{\mathrm{C}}=293(220) \mathrm{K}$ of $\mathrm{Gd}(\mathrm{Tb})$. Electron and phonon temperatures $\left(T_{e}\right.$ and $\left.T_{p}\right)$ enter via the differential equations of the well-established two-temperature model [48]. For the following, it is important that $\Delta E_{\mathrm{ex}}$ and $T_{e}$ are determined by evaluating the same experimental data, thus excluding errors in relative time delay. The rise of the electron temperature allows us to determine delay zero in the fit. With the delay fixed it is not possible to describe the exponential decay (dashed line in Fig. 4) of the exchange splitting within the M3TM (solid line). In this model, the change in $\Delta E_{\mathrm{ex}}$ is proportional to the phonon temperature $\left[T_{p}\right.$ in Eq. (1)] and is thus delayed from the heating of the electrons, since it takes time to heat the lattice. In contrast, in Fig. 4, the demagnetization is not delayed from the electronic temperature; $\Delta E_{\mathrm{ex}}$ drops at the same time that $T_{e}$ increases. We therefore conclude that in both $\mathrm{Gd}$ and $\mathrm{Tb}$ there exists besides EY electron-phonon scattering an additional microscopic process leading to ultrafast demagnetization, which follows the electronic rather than the lattice temperature. We also note that the fitted parameters $\tau_{R}$ of $9 \mathrm{ps}$ for $\mathrm{Tb}$ and 208 ps for Gd (solid line in Fig. 4) are incompatible with the time constants predicted by the M3TM [23], which is three times greater for $\mathrm{Tb}$ than for $\mathrm{Gd}$. This shortcoming may be corrected by a dynamic reduction of the exchange splitting, which leads to spin mixing [49] and can increase the spin-flip probability upon electron-phonon scattering [28]. 


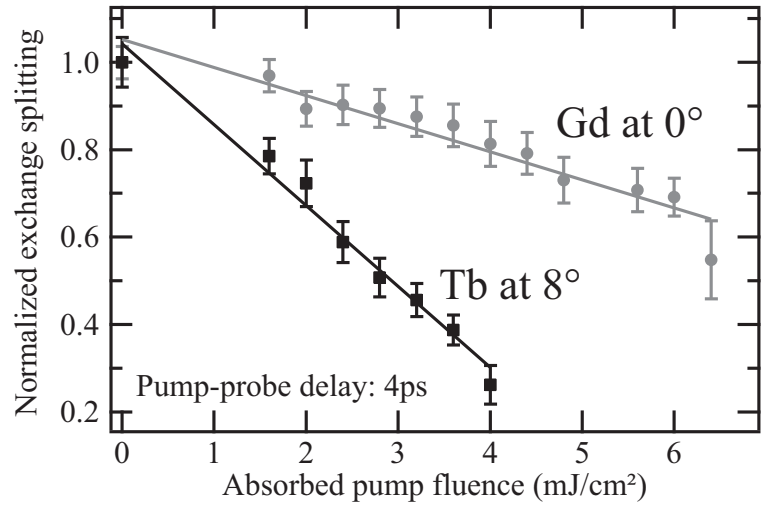

FIG. 5. Exchange splitting of Tb (squares) and Gd (circles) at 4 ps pump-probe delay vs absorbed pump fluence. At this delay, the exchange splitting reaches its minimum (see Fig. 3). Data for $\mathrm{Gd}$ and $\mathrm{Tb}$ were extracted at $0^{\circ}$ and $8^{\circ}$ emission angle, respectively. The relative error between absorbed fluences is within the symbol size.

Nevertheless, the EY scattering rate will still follow the lattice temperature.

As already reflected in the fitted time constants $\tau_{R}$ of the M3TM, the drop of the exchange splitting is faster for Tb than for Gd. From the data in Fig. 3, we see that the faster response of $\mathrm{Tb}$ results from a combination of several factors: In both materials, the minority spin band reacts faster than the majority spin band. Gd and $\mathrm{Tb}$ minority spin bands have similar decay times $(0.3$ versus $0.2 \mathrm{ps})$ but twice as large a shift in $\mathrm{Tb}$. In addition the Tb majority spin band responds faster than the Gd majority spin band ( 0.5 versus $0.9 \mathrm{ps}$ ) and without delay, while the Gd majority spin band shows a response with a delay of about 0.5 ps. Despite this complex response of the valence bands, the dynamics of the exchange splitting can be well described by a single exponential. From a fit to the data (dashed lines in Fig. 4), we obtain decay constants $\tau$ of $0.4 \pm 0.2$ ps for $\mathrm{Tb}$ and $1.1 \pm 0.2 \mathrm{ps}$ for $\mathrm{Gd}$. The time constant for Gd is in line with a MOKE measurement, which yielded a value of about $0.9 \mathrm{ps}$ for the same sample system $\mathrm{Gd} / \mathrm{W}(110)$ at a sample temperature of $100 \mathrm{~K}$, but for a significantly lower absorbed fluence of $1 \mathrm{~mJ} / \mathrm{cm}^{2}$ [6]. Compatible with our result, the time constant increases with the absorbed pump fluence [50]. The decay rate for $\mathrm{Tb}$ agrees with a MOKE measurement on a TbFe-based alloy, which shows an ultrafast decay component of $0.4 \mathrm{ps}$ at an excitation fluence of $4.4 \mathrm{~mJ} / \mathrm{cm}^{2}$ at $500 \mathrm{~nm}$. This response was attributed to the magnetization dynamics of Tb probed via the $4 f^{8} S_{7 / 2}$ multiplet component (see Fig. 1) [51].

What is more, the well-defined and comparable epitaxial films allow us to compare the material-specific fluence dependence of $\Delta E_{\mathrm{ex}}$. The drop of $\Delta E_{\mathrm{ex}}$ at 4 ps delay after optical excitation is depicted in Fig. 5. For Gd and Tb, the exchange splitting has been extracted at emission angles of $0^{\circ}$ and $8^{\circ}$, respectively. Compared to normal emission, the change of exchange splitting is slightly larger at $8^{\circ}$, since the $\mathrm{Tb}$ majority band has a smaller effective mass than its minority counterpart, but the band shift is nearly independent of the momentum $k$.

Between 1.5- and 40-ps delay we reach about the minimum of the exchange splitting. When $40 \mathrm{ps}$ have elapsed equilibrium among the different subsystems should be established. We therefore conclude that the values in Fig. 5 represent a true demagnetization of the valence electronic system. The reduction of the exchange splitting scales linearly with the absorbed pump fluence, but for the same fluence $\mathrm{Tb}$ shows a three times stronger change in $\Delta E_{\text {ex }}$ than $\mathrm{Gd}$. This is in line with a recent MOKE and XMCD study on spin-orbit coupling in GdTb alloys [19]. It calls for an additional mechanism of ultrafast demagnetization in $\mathrm{Tb}$.

\section{DISCUSSION}

\section{A. Dynamics of minority versus majority spin band}

Why does the minority spin band react faster than the majority spin band? The inelastic mean free path in the ARPES experiment is about two interlayer spacings. This can be judged, for example, from the intensity ratio between the $4 f$ surface and bulk components [42]. Our experiment is therefore very sensitive to nanoscale transport of hot carriers between surface and bulk, i.e., to superdiffusive (spin) currents [30]. Since transport occurs in the conduction bands, we can expect to observe its effects in the transient binding energy.

Both ARPES measurements [36] and ab initio bandstructure calculations [52] show that the minority spin band crosses the Fermi level along the $\Gamma-K$ direction of the Brillouin zone and lies above $E_{F}$ near the $K-H$ high-symmetry line. Thus a binding-energy shift of this partially unfilled band directly relates to transport of minority spin electrons. The band shifts to higher binding energy and becomes more occupied. Due to this band shift minority spin electrons accumulate. In addition, as seen from Figs. 1 and 2, the majority spin surface band shifts to lower binding energy above the Fermi level and becomes partially depopulated (see Fig. 3 in Refs. [7,37]). Consequently, majority spin electrons must leave the surface state. Together the band shifts imply a bidirectional flow of carriers but an unidirectional flow of spins. The net spin current can reduce the spin polarization at the surface and thus the magnetization. We note that we do not measure the full Fermi surface and can therefore not exclude compensating spin currents of opposite sign [53]. As transport sets in immediately upon laser excitation [30], we attribute the ultrafast response of the surface state and the minority spin band to superdiffusive spin transport between surface and bulk. This result is in line with the study of Melnikov et al. [54], who observed a drop of the Gd surface magnetization within the pump pulse duration by surface-sensitive second harmonic generation (SHG).

In contrast to the minority band, the ARPES measurements [36] and ab initio band-structure calculations [52] show that the majority spin band is fully occupied. The shift of this band can not affect its population. Nevertheless, spin-resolved ARPES measurements in thermal equilibrium show that the convergence of the Gd minority and majority spin bands is accompanied by a reduction of their spin polarization, which vanishes at $T_{\mathrm{C}}$ [49]. To change the spin polarization of the occupied majority spin band requires spin-flip processes. Since electron-phonon scattering increases with the number of excited phonons, the EY spin-flip scattering rate will depend on the lattice temperature $T_{p}$. It follows the decrease of 
the electronic temperature $T_{e}$ (see inset in Fig. 4) and thus increases more slowly than $T_{e}[47,48]$. This can qualitatively explain the slower response of the occupied majority spin band, which follows the increase of the lattice temperature.

The superdiffusive transport of nonequilibrium and hot electrons, i.e., of the tail of the Fermi Dirac distribution, sets in with laser excitation and will contribute to an ultrafast decay of the spin polarization of the valence electronic system (dashed line in Fig. 4). As this is not included in the M3TM, it fails to fully describe the initial drop of the exchange splitting (solid line in Fig. 4). To draw this conclusion, we need to know delay zero, which we extract from the fit of the electronic temperature (inset in Fig. 4). We therefore propose that spin currents between surface and bulk contribute to the ultrafast demagnetization of $\mathrm{Gd}$ and $\mathrm{Tb}$. This interpretation is in line with the nanoscale lateral spin transport seen in multilayers $[9,33,34]$ and inhomogeneous magnetic media [10].

\section{B. Dynamics of Gd versus $\mathbf{T b}$}

Considering only the valence electronic structure we would expect comparable magnetization dynamics for $\mathrm{Gd}$ and $\mathrm{Tb}$. While $\mathrm{Gd}$ is ferromagnetic up to the Curie temperature of $293 \mathrm{~K}$, Tb shows a helical antiferromagnetic phase between $T_{C}=220 \mathrm{~K}$ and the Néel temperature of $230 \mathrm{~K}$. These different magnetic structures have been related to the distinct Fermi surfaces of the lanthanides [55,56]. However, the peculiarities of the Gd and Tb electronic structure at the Fermi level should have little influence on the transport of hot carriers, which are optically excited up to $1.5 \mathrm{eV}$ above $E_{\mathrm{F}}$.

During the recovery of the magnetization, i.e., upon thermal cooling of the irradiated spot (30-500 ps), the band positions of both $\mathrm{Gd}$ and $\mathrm{Tb}$ follow the dependence seen in thermal equilibrium [41]. However, as described above, during laserinduced demagnetization (0-1.5 ps) $E \uparrow$ and $E \downarrow$ change independently with different time constants. In consequence, we observe different binding energies of the minority and majority spin bands for the same exchange splitting when comparing de- and re-magnetization [7]. This is illustrated in Fig. 6 by a plot of $E \uparrow$ and $E \downarrow$ against $\Delta E_{\text {ex }}$ for $\mathrm{Gd}$ (top) and $\mathrm{Tb}$ (bottom). The arrows in Fig. 6 indicate the direction of increasing pump-probe delay. The lower branches of the loops depict the binding energies during laser-driven demagnetization, while the upper branches depict the binding energies during thermal remagnetization. We observe two pathways because of the different decay constants $\tau$ for the $E \downarrow$ and $E \uparrow$ valence bands. The hysteresislike loops are more pronounced for $\mathrm{Gd}$ due to the different decay constants of the $\mathrm{Gd} \downarrow$ and $\mathrm{Gd} \uparrow$ valence bands and their temporal offset. In contrast, the decay constants of the $\mathrm{Tb} \downarrow$ and $\mathrm{Tb} \uparrow$ are more similar in size and the band dynamics start at the same delay (see Fig. 3). Consequently, the enclosed area per $\Delta E_{\mathrm{ex}}$ is smaller and the $\mathrm{Tb}$ loop is flatter than the Gd loop. From the hysteresislike loops in Fig. 6, we can conclude that optical excitation drives the $5 d$ and $4 f$ spin subsystems of both $\mathrm{Gd}$ and $\mathrm{Tb}$ out of equilibrium within the first $1.5 \mathrm{ps}$. Therefore the assumption that the $5 d$ spin system follows the $4 f$ spin system, which is reasonable under equilibrium conditions, may no longer be valid on the ultrafast time scale. We note that the recent first-principles study of Gd by Sandratskii [35] relies
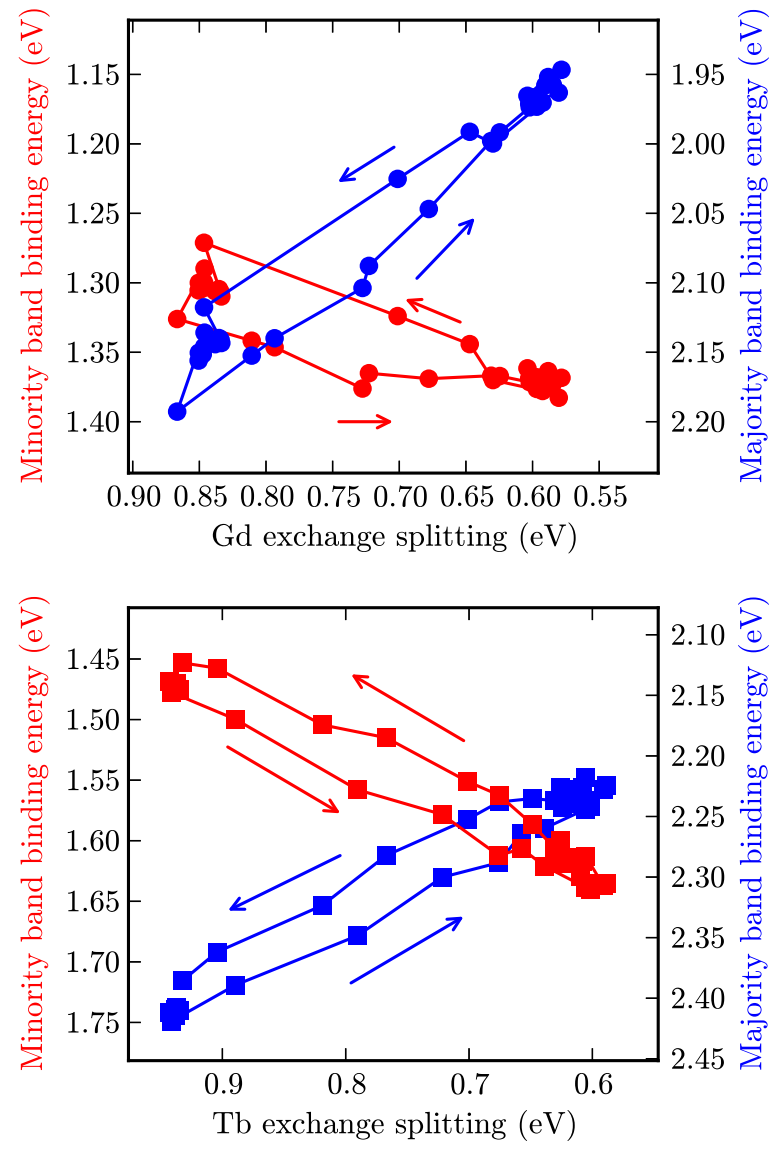

FIG. 6. (Color online) Laser-driven vs thermal effects in the minority (red) and majority (blue) spin components of the valence band of Gd (top) and $\mathrm{Tb}$ (bottom). During laser-driven demagnetization $(0-1.5 \mathrm{ps})$ the systems follow the lower path of each loop to smaller $\Delta E_{\mathrm{ex}}$. During thermal remagnetization $(\approx 30-500 \mathrm{ps})$ the systems cool and follow the upper branch to recover the exchange splitting. Arrows indicate the direction of increasing pump-probe delay.

on this assumption, which was put forward comparing XMCD and MOKE measurements [5]. In contrast, the nonequilibrium dynamics of the valence bands in $\mathrm{Gd}$ and $\mathrm{Tb}$ give first evidence for a decoupling of the valence and $4 f$ spin systems at early times after laser excitation. The hysteresis in Fig. 6 is somewhat stronger for $\mathrm{Gd}$ than for $\mathrm{Tb}$. Therefore we require an additional coupling between electron, phonon, and spin subsystems, which contributes to the equilibration of the $5 d$ and $4 f$ spin subsystems in $\mathrm{Tb}$.

During remagnetization (30-500 ps) electrons, lattice, $5 d$-spin, and $4 f$-spin subsystems are all close to thermal equilibrium. At that time, the exchange splitting $\Delta E_{\mathrm{ex}}$ reflects the temperature of the spin system via a relationship universal to the rare earths and close to the magnetization curves expected by mean-field theory, i.e., the exchange splitting scales with the $4 f$ moment $[57,58]$. If this is the case, about three times more angular momentum must be transferred to the $\mathrm{Tb} 4 f$ spin subsystem to explain the three times larger drop of the exchange splitting for the same absorbed pump fluence. The exchange interaction between the $5 d$ and $4 f$ electronic subsystems is too similar in $\mathrm{Gd}$ and $\mathrm{Tb}$ to explain such a large difference in angular momentum transfer. Reaching the 
minimum in $\Delta E_{\text {ex }}$ already after $\approx 1.5$ ps thus calls for ultrafast spin-lattice coupling in Tb. According to Hübner and Bennemann [59] the $4 f$-spin-lattice relaxation time $\tau_{\mathrm{SL}}$ is inversly proportional to the square of the anisotropy energy, which is related to the $4 f$ angular momentum $L$. In $\operatorname{Gd}(L \simeq 0)$, the anisotropy energy of $0.03 \mathrm{meV}$ is negligible but for $\mathrm{Tb}(L=3)$ it amounts to $10 \mathrm{meV}[60,61]$. Thus if $\tau_{\mathrm{SL}}$ is in the range of $10-40$ ps for Gd [5,47,62], it should be even smaller than the observed $\mathrm{Tb}$ time constant of $5 d$ demagnetization $\tau=0.54$ ps. We argue that $4 f$-spin-lattice coupling constitutes a sizable contribution to the demagnetization of Tb. Phonon-magnon scattering in $\mathrm{Tb}$ with an ultrafast time constant of about $0.3 \mathrm{ps}$ has also been found by Melnikov et al. in SHG [63].

This result is in contrast to the XMCD study of Gd and Tb by Wietstruk et al. [5], which revealed a two-step demagnetization with an ultrafast demagnetization time of $0.75 \pm 0.25$ ps identical for both systems and slower time constants of $40 \pm 10 \mathrm{ps}$ for $\mathrm{Gd}$ and $8 \pm 3 \mathrm{ps}$ for Tb. The long-time constants were attributed to spin-lattice coupling. While ARPES is sensitive to the valence bands and allows us to extract the exchange splitting, XMCD probes the $4 f$ magnetic moment. Moreover, the XMCD measurement is performed in transmission on thin, capped polycrystalline films. It is sensitive to the bulk magnetization and averages over fluences according to the absorption profile of the pump pulse. In contrast, the ARPES experiment probes the near surface region of an epitaxial film at a defined absorbed fluence. In both experiments, different quantities related to the sample magnetization are measured. In addition, transport effects can have a very different influence on the dynamics. This concerns not only spin currents and the ultrafast decay of the magnetization but also heat transport and the concomitant dynamics on the slower, picosecond time scale. The latter determines the recovery of the magnetization and is much faster in the crystalline samples (see Fig. 3 in the present paper and Fig. 3 in Ref. [5]). This may be the reason why in our ARPES experiment the dynamics of $\mathrm{Tb}$ and $\mathrm{Gd}$ are comparable on the longer, picosecond time scale, and the decay of the exchange splitting can be described by a single exponential. Here, we can only speculate that the biexponential decay of the Tb $4 f$ moment observed in XMCD follows the two-step behavior predicted by the M3TM for the rare-earth metals [23] and that the slow time constant of 8 ps does not reflect $\tau_{\mathrm{SL}}$.

Ultrafast $4 f$-spin-lattice coupling is likewise a suitable mechanism to speed up equilibration of the $5 d$ and $4 f$ spin subsystems via the phonon heat bath [14]. As a consequence of the different $4 f$-spin-lattice interaction in $\mathrm{Tb}$ and $\mathrm{Gd}$, the response times of the minority and majority spin valence bands will be more like in $\mathrm{Tb}$ than in $\mathrm{Gd}$, as observed in our ARPES experiment.

\section{SUMMARY}

To conclude, superdiffusive spin transport and ElliottYafet-type spin-flip scattering are reflected in the dynamics of different spin components of the electronic valence bands of $\mathrm{Gd}$ and $\mathrm{Tb}$. The drop of the exchange splitting in $\mathrm{Tb}$ is about twice as fast and three times stronger than in Gd, suggesting an additional contribution from $4 f$-spin-lattice coupling. These contributions are particularly important for alloys, where upon hybridization of valence and $4 f$ states, spin-lattice coupling and transport on the nanoscale can contribute to the magnetization dynamics and drive ultrafast magnetic switching.

\section{ACKNOWLEDGMENTS}

We wish to thank Karel Carva, Peter M. Oppeneer, and Leonid M. Sandratskii for valuable discussions. We gratefully acknowledge funding from the Leibniz Graduate School Dynamics in New Light, the Helmholtz Virtual Institute Dynamic Pathways in Multidimensional Landscapes, and the Deutsche Forschungsgemeinschaft through Grant WE2037/4-1.
[1] C. Stamm, T. Kachel, N. Pontius, R. Mitzner, T. Quast, K. Holldack, S. Khan, C. Lupulescu, E. F. Aziz, M. Wietstruk, H. A. Dürr, and W. Eberhardt, Nat. Mater. 6, 740 (2007).

[2] J.-Y. Bigot, M. Vomir, and E. Beaurepaire, Nat. Phys. 5, 515 (2009).

[3] A. Kirilyuk, A. V. Kimel, and T. Rasing, Rev. Mod. Phys. 82, 2731 (2010).

[4] C. Boeglin, E. Beaurepaire, V. Halté, V. López-Flores, C. Stamm, N. Pontius, H. A. Dürr, and J.-Y. Bigot, Nature (London) 465, 458 (2010).

[5] M. Wietstruk, A. Melnikov, C. Stamm, T. Kachel, N. Pontius, M. Sultan, C. Gahl, M. Weinelt, H. A. Dürr, and U. Bovensiepen, Phys. Rev. Lett. 106, 127401 (2011).

[6] M. Sultan, U. Atxitia, A. Melnikov, O. Chubykalo-Fesenko, and U. Bovensiepen, Phys. Rev. B 85, 184407 (2012).

[7] R. Carley, K. Döbrich, B. Frietsch, C. Gahl, M. Teichmann, O. Schwarzkopf, P. Wernet, and M. Weinelt, Phys. Rev. Lett. 109, 057401 (2012).
[8] I. Radu, K. Vahaplar, C. Stamm, T. Kachel, N. Pontius, H. A. Dürr, T. A. Ostler, J. Barker, R. F. L. Evans, R. W. Chantrell, A. Tsukamoto, A. Itoh, A. Kirilyuk, T. Rasing, and A. V. Kimel, Nature (London) 472, 205 (2011).

[9] D. Rudolf, C. La-O-Vorakiat, M. Battiato, R. Adam, J. M. Shaw, E. Turgut, P. Maldonado, S. Mathias, P. Grychtol, H. T. Nembach, T. J. Silva, M. Aeschlimann, H. C. Kapteyn, M. M. Murnane, C. M. Schneider, and P. M. Oppeneer, Nat. Commun. 3, 1031 (2012).

[10] C. E. Graves, A. H. Reid, T. Wang, B. Wu, S. de Jong, K. Vahaplar, I. Radu, D. P. Bernstein, M. Messerschmidt, L. Müller, R. Coffee, M. Bionta, S. W. Epp, R. Hartmann, N. Kimmel, G. Hauser, A. Hartmann, P. Holl, H. Gorke, J. H. Mentink, A. Tsukamoto, A. Fognini, J. J. Turner, W. F. Schlotter, D. Rolles, H. Soltau, L. Strüder, Y. Acremann, A. V. Kimel, A. Kirilyuk, T. Rasing, J. Stöhr, A. O. Scherz, and H. A. Dürr, Nat. Mater. 12, 293 (2013).

[11] S. Mangin, M. Gottwald, C.-H. Lambert, D. Steil, V. Uhlír, L. Pang, M. Hehn, S. Alebrand, M. Cinchetti, G. Malinowski, 
Y. Fainman, M. Aeschlimann, and E. E. Fullerton, Nat. Mater. 13, 286 (2014).

[12] N. Bergeard, V. López-Flores, V. Halté, M. Hehn, C. Stamm, N. Pontius, E. Beaurepaire, and C. Boeglin, Nat. Commun. 5, 3466 (2014).

[13] T. A. Ostler, J. Barker, R. F. L. Evans, R. W. Chantrell, U. Atxitia, O. Chubykalo-Fesenko, S. El Moussaoui, L. Le Guyader, E. Mengotti, L. J. Heyderman, F. Nolting, A. Tsukamoto, A. Itoh, D. Afanasiev, B. A. Ivanov, A. M. Kalashnikova, K. Vahaplar, J. Mentink, A. Kirilyuk, T. Rasing, and A. V. Kimel, Nat. Commun. 3, 666 (2012).

[14] E. Beaurepaire, J.-C. Merle, A. Daunois, and J.-Y. Bigot, Phys. Rev. Lett. 76, 4250 (1996).

[15] J. Hohlfeld, E. Matthias, R. Knorren, and K. H. Bennemann, Phys. Rev. Lett. 78, 4861 (1997).

[16] H.-S. Rhie, H. A. Dürr, and W. Eberhardt, Phys. Rev. Lett. 90, 247201 (2003).

[17] C. Stamm, N. Pontius, T. Kachel, M. Wietstruk, and H. A. Dürr, Phys. Rev. B 81, 104425 (2010).

[18] T. Roth, A. J. Schellekens, S. Alebrand, O. Schmitt, D. Steil, B. Koopmans, M. Cinchetti, and M. Aeschlimann, Phys. Rev. X 2, 021006 (2012).

[19] A. Eschenlohr, M. Sultan, A. Melnikov, N. Bergeard, J. Wieczorek, T. Kachel, C. Stamm, and U. Bovensiepen, Phys. Rev. B 89, 214423 (2014).

[20] J. H. Mentink, J. Hellsvik, D. V. Afanasiev, B. A. Ivanov, A. Kirilyuk, A. V. Kimel, O. Eriksson, M. I. Katsnelson, and T. Rasing, Phys. Rev. Lett. 108, 057202 (2012).

[21] S. Wienholdt, D. Hinzke, K. Carva, P. M. Oppeneer, and U. Nowak, Phys. Rev. B 88, 020406 (2013).

[22] A. Kirilyuk, A. V. Kimel, and T. Rasing, Rep. Prog. Phys. 76, 026501 (2013).

[23] B. Koopmans, G. Malinowski, F. Dalla Longa, D. Steiauf, M. Fähnle, T. Roth, C. Cinchetti, and M. Aeschlimann, Nat. Mater. 9, 259 (2010).

[24] K. C. Kuiper, T. Roth, A. J. Schellekens, O. Schmitt, B. Koopmans, M. Cinchetti, and M. Aeschlimann, Appl. Phys. Lett. 105, 202402 (2014).

[25] E. Carpene, E. Mancini, C. Dallera, M. Brenna, E. Puppin, and S. De Silvestri, Phys. Rev. B 78, 174422 (2008).

[26] M. Krauß, T. Roth, S. Alebrand, D. Steil, M. Cinchetti, M. Aeschlimann, and H. C. Schneider, Phys. Rev. B 80, 180407 (2009).

[27] K. Carva, M. Battiato, and P. M. Oppeneer, Phys. Rev. Lett. 107, 207201 (2011).

[28] B. Y. Mueller, A. Baral, S. Vollmar, M. Cinchetti, M. Aeschlimann, H. C. Schneider, and B. Rethfeld, Phys. Rev. Lett. 111, 167204 (2013).

[29] C. Illg, M. Haag, and M. Fähnle, Phys. Rev. B 88, 214404 (2013).

[30] M. Battiato, K. Carva, and P. M. Oppeneer, Phys. Rev. Lett. 105, 027203 (2010).

[31] V. P. Zhukov, E. V. Chulkov, and P. M. Echenique, Phys. Rev. Lett. 93, 096401 (2004).

[32] A. Eschenlohr, M. Battiato, P. Maldonado, N. Pontius, T. Kachel, K. Holldack, R. Mitzner, A. Föhlisch, P. M. Oppeneer, and C. Stamm, Nat. Mater. 12, 332 (2013).

[33] B. Vodungbo, J. Gautier, G. Lambert, A. B. Sardinha, M. Lozano, S. Sebban, M. Ducousso, W. Boutu, K. Li, B. Tudu, M. Tortarolo, R. Hawaldar, R. Delaunay, V. López-Flores,
J. Arabski, C. Boeglin, H. Merdji, and P. Z. J. Lüning, Nat. Comm. 3, 999 (2012).

[34] B. Pfau, S. Schaffert, L. Müller, C. Gutt, A. Al-Shemmary, F. Büttner, R. Delaunay, S. Düsterer, S. Flewett, R. Frömter, J. Geilhufe, E. Guehrs, C. M. Günther, R. Hawaldar, M. Hille, N. Jaouen, A. Kobs, K. Li, J. Mohanty, H. Redlin, W. F. Schlotter, D. Stickler, R. Treusch, B. Vodungbo, M. Kläui, H. P. Oepen, J. Lüning, G. Grübel, and S. Eisebitt, Nat. Commun. 3, 1100 (2012).

[35] L. M. Sandratskii, Phys. Rev. B 90, 184406 (2014).

[36] K. M. Döbrich, G. Bihlmayer, K. Starke, J. E. Prieto, K. Rossnagel, H. Koh, E. Rotenberg, S. Blügel, and G. Kaindl, Phys. Rev. B 76, 035123 (2007).

[37] M. Lisowski, P. A. Loukakos, A. Melnikov, I. Radu, L. Ungureanu, M. Wolf, and U. Bovensiepen, Phys. Rev. Lett. 95, 137402 (2005).

[38] B. Frietsch, R. Carley, K. Döbrich, C. Gahl, M. Teichmann, O. Schwarzkopf, P. Wernet, and M. Weinelt, Rev. Sci. Inst. 84, 075106 (2013).

[39] B. Kim, A. B. Andrews, J. L. Erskine, K. J. Kim, and B. N. Harmon, Phys. Rev. Lett. 68, 1931 (1992).

[40] M. Bode, M. Getzlaff, A. Kubetzka, R. Pascal, O. Pietzsch, and R. Wiesendanger, Phys. Rev. Lett. 83, 3017 (1999).

[41] C. Schüßler-Langeheine, Ph.D. thesis, Freie Universität Berlin, 1999 .

[42] K. Starke, L. Baumgarten, E. Arenholz, E. Navas, and G. Kaindl, Phys. Rev. B 50, 1317 (1994).

[43] U. Bovensiepen, J. Phys.: Cond. Matt. 19, 083201 (2007).

[44] K. Carva (private communication).

[45] B. Andres, Diploma thesis, Freie Universität Berlin, 2011.

[46] V. S. Fomenko, Powder Metall. Met. Ceram. 33, 85 (1995).

[47] A. Melnikov, H. Prima-Garcia, M. Lisowski, T. Gießel, R. Weber, R. Schmidt, C. Gahl, N. M. Bulgakova, U. Bovensiepen, and M. Weinelt, Phys. Rev. Lett. 100, 107202 (2008).

[48] S. I. Anisimov, B. L. Kapeliovich, and T. L. Perel'man, Zh. Eksp. Teor. Fiz 66, 776 (1974) [Sov. Phys.-JETP 39, 375 (1974)].

[49] K. Maiti, M. C. Malagoli, A. Dallmeyer, and C. Carbone, Phys. Rev. Lett. 88, 167205 (2002).

[50] M. Sultan, A. Melnikov, and U. Bovensiepen, Phys. Status Solidi B 248, 2323 (2011).

[51] A. R. Khorsand, M. Savoini, A. Kirilyuk, A. V. Kimel, A. Tsukamoto, A. Itoh, and T. Rasing, Phys. Rev. Lett. 110, 107205 (2013).

[52] P. Kurz, G. Bihlmayer, and S. Blügel, J. Phys.: Condens. Matter 14, 6353 (2002).

[53] A. Melnikov, I. Razdolski, T. O. Wehling, E. T. Papaioannou, V. Roddatis, P. Fumagalli, O. Aktsipetrov, A. I. Lichtenstein, and U. Bovensiepen, Phys. Rev. Lett. 107, 076601 (2011).

[54] A. Melnikov, I. Radu, U. Bovensiepen, O. Krupin, K. Starke, E. Matthias, and M. Wolf, Phys. Rev. Lett. 91, 227403 (2003).

[55] I. D. Hughes, M. Däne, A. Ernst, W. Hergert, M. Lüders, J. Poulter, J. B. Staunton, A. Svane, Z. Szotek, and W. M. Temmerman, Nature (London) 446, 650 (2007).

[56] K. M. Döbrich, A. Bostwick, J. L. McChesney, K. Rossnagel, E. Rotenberg, and G. Kaindl, Phys. Rev. Lett. 104, 246401 (2010).

[57] R. Ahuja, S. Auluck, B. Johansson, and M. S. S. Brooks, Phys. Rev. B 50, 5147 (1994). 
[58] C. Schüßler-Langeheine, E. Weschke, C. Mazumdar, R. Meier, A. Y. Grigoriev, G. Kaindl, C. Sutter, D. Abernathy, G. Grübel, and M. Richter, Phys. Rev. Lett. 84, 5624 (2000).

[59] W. Hübner and K. H. Bennemann, Phys. Rev. B 53, 3422 (1996).

[60] J. J. Rhyne and A. E. Clark, J. Appl. Phys. 38, 1379 (1967).
[61] S. Abdelouahed and M. Alouani, Phys. Rev. B 79, 054406 (2009).

[62] A. Vaterlaus, T. Beutler, and F. Meier, Phys. Rev. Lett. 67, 3314 (1991).

[63] A. Melnikov, A. Povolotskiy, and U. Bovensiepen, Phys. Rev. Lett. 100, 247401 (2008). 